\title{
A multidimensional evaluation of the perception and annoyance caused by railway induced groundborne vibration
}

\author{
Woodcock, J \\ j.s.woodcock@salford.ac.uk \\ Moorhouse, AT \\ a.t.moorhouse@salford.ac.uk \\ Waddington, DC \\ d.c.waddington@salford.ac.uk
}

May 26, 2015

\begin{abstract}
This paper presents a study investigating seated subjects' perception of railway induced groundborne vibration in the vertical direction. Previous studies into the perception of railway induced vibration have treated this phenomenon as a unidimensional problem. The aim of the study detailed in this paper is to determine if the perception of railway induced groundborne vibration is multidimensional in nature and if the resulting perceptual dimensions can be related to a measure of annoyance. Twenty-one subjects took part in paired comparison tests of similarity and annoyance. These tests were conducted using fourteen measured vibration stimuli selected to be representative of groundborne vibration induced by railway activities in the United Kingdom. Through multidimensional scaling analysis, it is shown that the perception of railway induced vibration is dependent on up to four perceptual dimensions. These dimensions relate to energy in the $16 \mathrm{~Hz} 1 / 3$ octave band $\left(a_{16 \mathrm{~Hz}}\right)$, energy in the $32 \mathrm{~Hz}$ $1 / 3$ octave band $\left(a_{32 \mathrm{~Hz}}\right)$, the duration of the train passage $\left(T_{10 \mathrm{~dB}}\right)$, and the modulation frequency of the envelope of the signal $\left(f_{\text {mod }}\right)$. These perceptual dimensions are shown to be related to single figure Perceived Annoyance Ratings $(A)$ by the following relationship: $A=-0.40+4.57 a_{16 H z}+3.18 a_{32 H z}+0.02 T_{10 d B}+0.02 f_{\text {mod }}$. Finally, the single figure Perceived Annoyance Ratings are related to categorical ratings of annoyance via a logistic regression model. These findings
\end{abstract}


confirm the hypothesis that the perception of complex vibration stimuli is multidimensional and can be described by a small number of perceptual dimensions.

\section{Introduction}

Noise and vibration from railway operations can be a source of significant disturbance to residents living close to railway lines. With current EU policy focussed towards a modal shift of goods traffic from road to rail [1] and the building of high speed lines in a number of countries, an understanding is needed of the human response to railway induced vibration and noise if a concomitant increase in annoyance for residents living in close vicinity of railway lines is to be avoided.

In the past two decades, a number of field studies have been carried out in Europe, North America and Canada, and Japan which have developed exposure-response relationships for the prediction of the community response to railway induced vibration [2, 3, 4, 5, 6, 7]. A common trend in these studies is the relatively low amount of variance explained by the resulting exposure-response relationships. As is the case for environmental noise, this may be due to both factors not related to the vibration exposure [8, 9] and an inadequacy in the single figure vibration exposure descriptors to describe human response. Although a substantial amount of fundamental psychophysical work has been conducted for the human response to vibration such as investigations into subjective magnitude [10, perception thresholds [11, and equal comfort contours [12, there is a lack of understanding regarding the perception of complex vibration from real sources.

There have been a limited number of laboratory studies into annoyance due to railway vibration and noise. A fourth power relationship has been found in a study to determine the relationship between the magnitude of railway induced vibration and the number of events with respect to annoyance [13]. A similar result was found in an investigation into the perception of vertical mechanical shocks [14]. The fourth power relationship suggested by these studies is part of the justification for the vibration dose value descriptor that is recommended in international and British standards. In a laboratory study to investigate the subjective response to combined noise and vibration exposure from railways [15, 16], the magnitude of noise ex- 
posure was found to have a significant effect on the judgment of annoyance caused by vibration but no significant effect of vibration exposure was found on the judgment of annoyance caused by exposure to noise. In a subsequent study by the same authors [17], a relationship showing the subjective equivalence of railway noise and vibration was derived. In a more recent study into the combined effects of railway induced vibration and noise [18, it was also found that vibration did not influence noise annoyance, but that total annoyance caused by combined noise and vibration was considerably greater than the annoyance caused by noise alone. However, in all of these studies the perception of vibration has been treated as a unidimensional phenomenon.

The aim of the study presented in this paper is to investigate the dimensions of the perception of railway induced groundborne vibration and how these perceptual dimensions relate to annoyance. In areas of perceptual acoustics such as musical timbre [19], soundscapes research [20], and product sound quality [21], research often focusses on determining the perceptual dimensions which underlie the perception of a given set of sounds. In the case of product sound quality, these perceptual dimensions have been used to develop models that can be used to predict the perceived quality of a product based on objective acoustic features of the product sound. If a similar approach can be taken towards the perception of vibration from environmental sources, it may be possible to develop models to predict perceived annoyance based upon objective features of a measured vibration signal.

The multidimensional nature of sound perception is highlighted by the rich vocabulary available for the description of auditory perception. For example, frequency characteristics of a sound can be described as "bright", "sharp", or "dull"; amplitude characteristics can be described as "loud" or "quiet"; and temporal characteristics can be described "fluctuating", "peaky", or "undulating". In comparison to the perception of auditory stimuli, the vocabulary at our disposal for describing the perception of vibratory stimuli is rather limited. This might be taken to suggest that the acuity of human perception of vibration is less than that of the perception of sound. Nevertheless, research conducted in the automotive industry has suggested that the perception of vibration is a multidimensional phenomenon [22]. Therefore, a major aim of the work presented in this paper is to determine if the perception of railway induced groundborne vibration 
is multidimensional in nature.

Much of the previous research into the perception of whole body vibration has been in the form of ranking or magnitude estimation tasks conduced in a laboratory setting using artificial signals such as pure sine excitation as stimuli. Although research of this sort provides a valuable insight into psychophysical aspects of vibration perception such as perception thresholds and subjective magnitude, these subjective test methodologies impose limitations on the researcher, namely, the perceptual dimension or dimensions of interest must be determined a priori [23]. If the underlying perceptual dimensions of a certain stimulus type are unknown, then it is possible that psychologically relevant dimensions will be unaccounted for in the models and metrics used to describe the human response these stimuli. Therefore, little is known regarding the nature of the psychologically relevant dimensions which determine the perception of whole body vibration. It is hypothesised that the perception of vibration from railway activities can be described by a small number of perceptual dimensions and that these dimensions can be related to objective features of the vibration stimuli. It is further hypothesised that, if an understanding can be gained of the perceptual structure of a set of complex vibration stimuli, models can be developed which relate the salient perceptual dimensions to some measure of response, in this case annoyance.

In this paper, the methods of paired comparisons and multidimensional scaling analysis are employed to investigate the perceptual dimensions underlying the perception of railway induced vibration. The study focusses on seated subjects' perception of vertical railway induced vibration. As the sensitivity of the human body to vibration differs considerably depending upon the orientation and posture of the subject, the results of this study do not necessarily apply to the perception of subjects in other orientations. The social survey described in [6] asked 932 subjects through with surfaces they were able to perceive railway induced vibration. $70.5 \%$ of the respondents were able to feel vibration through the floor, $63.6 \%$ through the bed, $51.3 \%$ through a chair, $29.4 \%$ through touching surfaces with their hands, and $11.7 \%$ through other sources. This indicates that a large proportion of people who experience railway induced vibration in residential environments experience it in a seated posture.

Figure 1 illustrates the basic principles of multidimensional scaling anal- 
ysis in the context of vibration perception. In this figure the subject is exposed to four different railway vibration events in all possible pairs. The subject is asked to rate the degree of similarity between the pairs of vibration events on a continuous scale ranging between 0 and 1. It is assumed that there exists a latent perceptual structure which allows the subject to make comparisons between the vibration stimuli, that this structure consists of a finite number of perceptual dimensions, and that the subject's similarity ratings represent a comparison between stimuli based upon this psychological structure. The similarity matrix resulting from these judgements is then submitted to multidimensional scaling analysis which attempts to create a mapping of the stimuli in a low-dimensional space. It is then assumed that each of the dimensions in the multidimensional scaling configuration relate to a dimension in the psychological structure which the subject uses to make comparisons between the stimuli. By finding objective features of the vibration stimuli which correlate to these dimensions, an understanding can be gained as to how objective features of the vibration stimuli influence perception. The methods of paired comparisons and multidimensional scaling have been used extensively in areas such as the perception of musical timbre [19, 24] the perception of concert hall quality [25], and product sound quality [26, 27, 28, 29].

In the following section, the methods used in the study are outlined. Following this, the results of the study are presented and discussed. Finally conclusions are drawn and recommendations for future work are made.

\section{Methods}

\section{$2.1 \quad$ Ethical considerations}

The research presented in this paper was approved by the University of Salford Ethics Committee.

\subsection{Selection of stimuli}

A database of over 64,500 measured railway vibration signals, collected as part of a large scale field survey conducted in the United Kingdom [30], were available as potential stimuli for the subjective tests described in this paper. These signals were taken from 149 24-hour measurements that spanned 


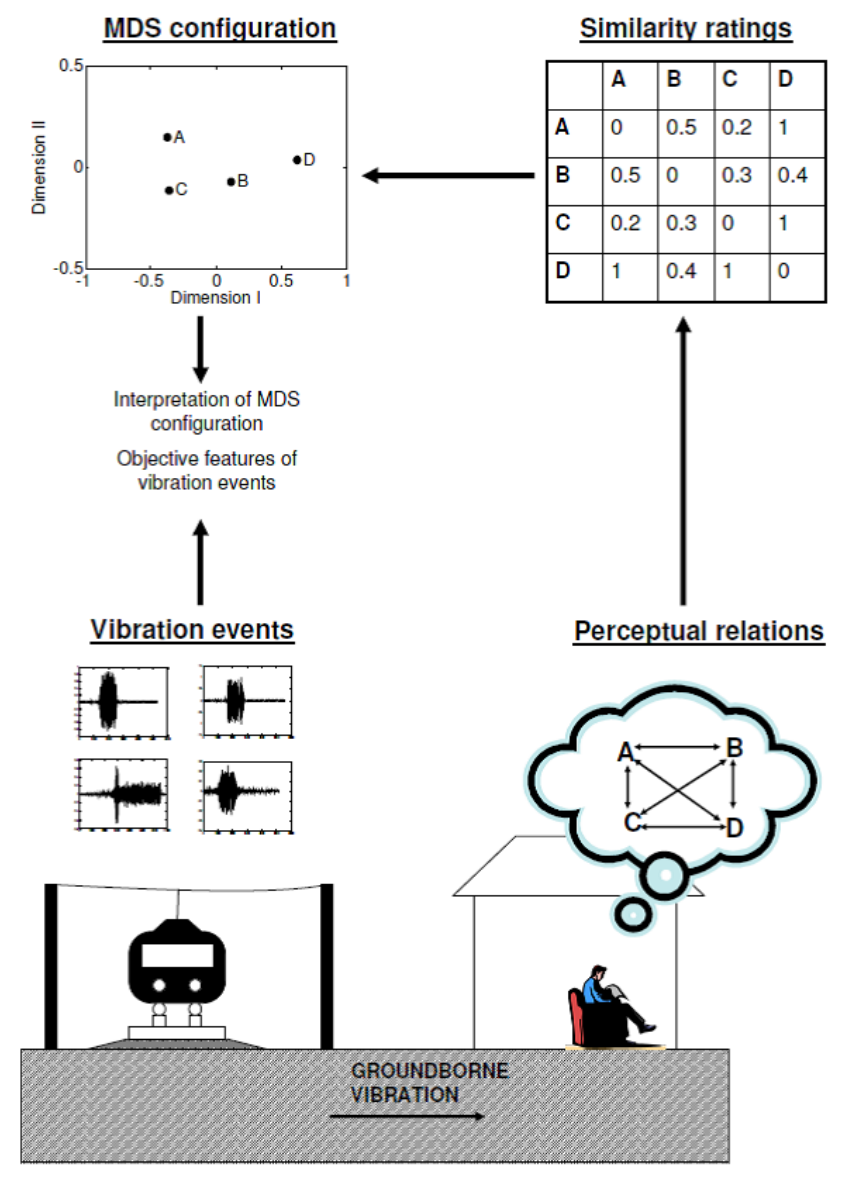

Figure 1: Illustration of the basic principles of multidimensional scaling as it relates to the perception of railway induced vibration. 
around $200 \mathrm{~km}$ of the West Coast Mainline in the United Kingdom. As the database of measured railway signals were from unattended measurements, information such as train type and speed were unavailable. Due to the number of available test signals, a stimulus reduction was required to generate a representative set of stimuli which could be practicably used in a subjective test. The stimuli selection routine was designed to result in a representative subset of the types, speeds, and conditions of trains running on this stretch of line.

As an initial step, any of the available train signals with a $W_{k}$ weighted peak magnitude less than $0.015 \mathrm{~m} / \mathrm{s}^{2}$ was excluded as a potential test stimulus. This step was taken as, according to ISO 2331-1:1997 [31], this magnitude is the median vibration perception threshold for healthy human subjects. Although the applicability of this laboratory derived perception threshold to vibration under field conditions is not clear, this appeared to be the logical first step in the reduction of the stimulus set. Following this initial reduction, 14,143 train signals remained as potential stimuli.

For the remaining signals, a number of objective descriptors for frequency, energy, and temporal characteristics of each train signal were calculated. The descriptors of energy calculated were rms acceleration and the vibration dose value [VDV, see equation (1)] as defined in ISO 2331-1:1997 [31. Spectral centroid was calculated as a descriptor of the frequency content of each train signal. To describe temporal aspects of the train signals the crest factor, kurtosis, duration defined by the $3 d B$ and $10 d B$ down points of the signal envelope, the modulation depth, and modulation frequency were calculated.

$$
V D V=\sqrt[4]{\int_{0}^{T} a_{w}^{4}(t) d t}
$$

The modulation depth was defined as the average difference between the maxima and minima of the signal envelope expressed in decibels. Modulation frequency was defined as one over the average period between the maxima of the signal envelope. These parameters were evaluated in the portion of the signal between the $10 d B$ up and down points.

A principal component analysis was then conducted on a matrix of these descriptors. The first four recovered principal components were found to describe almost $80 \%$ of the explained variance in the descriptor space. To 
select the stimulus set for the subjective test, each of the four recovered principal components was divided into four equal areas and a single train signal was randomly selected from each area. Upon investigation of the generated stimulus set, it was discovered that a number of the signals at the positive extreme of the first principal component were spurious events such as footfalls. After removing these spurious events from the analysis, a final stimulus set of fourteen train signals was arrived at.

\section{$2.3 \quad$ Test rig}

A test rig was built which was designed to be capable of the faithful and repeatable reproduction of measured vibration signals. The rig consists of an electrodynamic shaker coupled to a frame consisting of a table supported on springs and linear guides with a chair with a cushioned seated rigidly fixed to the table. As with any vibration reproduction system, there is some uncertainty in the vibration exposure for different subjects due to variations in mass across the subject group. The shaker used in this setup was a Derritron VP-85 powered by a 6000 Watt TW6000 amplifier. Broadband noise produced by the amplifier in the laboratory was sufficient to mask sounds generated by the shaker and the shaker table frame. Consequently, subjects were not exposed to sound that was correlated with the vibration stimuli. As the manufacturer stated maximum static load of the shaker is $35 \mathrm{~kg}$, the spring supports were included in the test rig to ensure that the shaker was not loaded with the full mass of a test subject. The linear guides were included in the design to constrain the movement of the shaker table to the vertical direction.

\subsection{Test design}

Twenty-one subjects (20 males and 1 female, mean age $=32.9$, sd $=8.9$ ) participated in the subjective tests described in this paper. This is a similar number of subjects as other studies into the perception of railway induced vibration [16, 17, 18]. As full paired comparison tests using stimuli of long duration can be prohibitively lengthy, incomplete paired comparison test designs were utilised to reduce the length of the paired comparison tests. It has been shown by Spence and Domoney [32] that many of the pairs tested in a complete paired comparison design lead to essentially redundant data. 
Spence and Domoney investigated data redundancy in paired comparison tests by conducting Monte-Carlo simulations to determine the influence of incomplete paired comparison data upon non-metric scaling configurations. Through these simulations it was found that a reasonable reconstruction of the resulting multidimensional scaling configuration could be arrived at using only $30 \%$ of the original data. An incomplete paired comparison test was designed for each of the subjects taking part in the test. Depending on the incomplete design, the number of pairs which each subject had to rate varied between 42 and 56 . For 14 stimuli a full paired comparison test would require each subject to judge 91 pairs of stimuli, therefore the incomplete test designs used in this paper resulted in perceptual tests with between $46 \%$ and $62 \%$ of the number of pairs which would have been required if using a full paired comparison design.

Prior to the start of the subjective test, subjects were provided with written and verbal instructions of their task. Following this, they were asked to sit comfortably on the chair of the test rig with their feet supported by a stationary footrest. Once sat in a comfortable position, the subject was asked to maintain their posture as far as possible throughout the test. Subjects were then given the opportunity to familiarise themselves with the test interface, which was presented via a laptop computer, via five trial pairs of vibration stimuli. Once the subject had familiarised themselves with the test interface, they were given the opportunity to feel each of the fourteen vibration stimuli used in the test to familiarise themselves with the group of stimuli.

Subjects were presented with pairs of vibration stimuli separated by 1 $\mathrm{s}$ and ordered according to an incomplete cyclic design. The start of each stimulus was marked via a $0.5 \mathrm{~s}$ beep generated by a loudspeaker. Subjects were asked to make two judgements upon each pair of vibration stimuli:

1. Which of the trains would bother, disturb, or annoy you most if you felt them in your home?

2. How similar do you perceive the pair of vibrations to be?

The responses to both of these questions were recorded via continuous sliders and coded -0.5 to 0.5 for question 1 ) and 0 to 1 for question 2). Subjects were allowed to feel each pair of vibration stimuli as many times as they wished. 
To measure annoyance on an absolute scale, subjects were exposed to each of the vibration stimuli individually and asked the following question:

If you were in your own home how bothered, disturbed, or annoyed would you be by this vibration?

Responses to this question were recorded on a five-point semantic scale with the category labels "Not at all", "Slightly", "Moderately", "Very", and "Extremely". This part of the test took place immediately after the paired comparison judgements detailed in the previous section.

\subsection{Data analysis}

Analysis of the data measured in the perceptual testing consists of three main steps: i) the similarity data is analysed using multidimensional scaling (MDS) analysis to derive a perceptual space; ii) objective correlates to the perceptual dimensions are explored using correlation analysis; iii) models for relative annoyance are built via linear regression models using single figure annoyance ratings calculated from the paired comparison of annoyance data and the objective metrics identified in ii); vi) models for absolute annoyance are built via logistic regression models using the single figure perceived annoyance ratings and the categorical annoyance ratings.

\subsubsection{Multidimensional scaling}

The general procedure for MDS is to find a configuration of points in low dimensional Euclidean space where distances between points $\left(d_{i, j}\right)$ are approximately equal to $f\left(\delta_{i, j}\right)$ where $f$ is a parametric monotonic function and $\delta_{i, j}$ are measured pairwise distances. This is commonly achieved by fitting the matrix of distances $d_{i, j}$ by least squares or eigendecomposition to $f\left(\delta_{i, j}\right)$. For example, a configuration may be sought which minimises the loss function given in equation (2) where the parameters of the function $f$ are to be estimated [33].

$$
\sqrt{\frac{\sum_{i, j}\left(d_{i, j}-f\left(\delta_{i, j}\right)\right)^{2}}{\sum_{i, j}\left(d_{i, j}\right)^{2}}}
$$

where $d_{i, j}$ are the reproduced distances, and $\delta_{i, j}$ are measured dissimilarities. 
The form of the function $f$ is largely dependent on the measurement level of the input data $\left\{\delta_{i, j}\right\}$. If the data to be analysed by MDS are on the interval or ratio scale, metric multidimensional scaling can be used. For metric multidimensional scaling, a constraint on the function $f$ is imposed such that $f$ must be continuous and monotonic. If the data to be analysed are on the ordinal scale, non-metric multidimensional scaling may be a more appropriate model. In non-metric MDS, a relaxation on the constraints imposed on the function $f$ is introduced such that $f$ may be a non-parametric monotonic function. In contrast to metric scaling which attempts to find a configuration of points in low-dimensional Euclidean space which preserves the measured distances between objects, non-metric multidimensional scaling attempts to provide a configuration in which the distances between points preserves the rank order of judged dissimilarities. In-depth discussions of metric and nonmetric MDS models can be found in a number of publications [33, 34, 35].

\subsubsection{Correlation}

Relationships between the dimensions revealed through the multidimensional scaling analysis and objective features of the vibration exposure are assessed using Pearson's correlation coefficient. Pearson's correlation coefficient provides a measure of the linear dependence between two variables. The resulting values range from -1 to 1 with -1 indicating a perfect negative correlation, 0 indicating no correlation, and 1 indicating a perfect positive correlation. For two variables $X$ and $Y$ with observed values $x_{i}$ and $y_{i}$ for $i=1,2, \ldots, n$, Pearson's correlations coefficient can be calculated using equation (3).

$$
\rho_{x y}=\frac{\sum\left(x_{i}-\bar{x}\right)\left(y_{i}-\bar{y}\right)}{\sqrt{\sum\left(x_{i}-\bar{x}\right)^{2} \sum\left(y_{i}-\bar{y}\right)^{2}}}
$$

where $\bar{x}$ is the mean of all $x_{i}$ s of $X$ and where $\bar{y}$ is the mean of all $y_{i}$ s of $Y$.

\subsubsection{Regression models}

Models relating objective features of the stimuli to the measured subjective responses have been derived using regression models. Multiple linear regression is a technique that can be used to study the relationship between a response variable $Y$ and predictor variables $\mathbf{X}$ [36]. In multiple linear regression, an equation is estimated from observed data that expresses a response 
variable as a linear function of a number of predictor variables as shown in equation (4)

$$
Y=\mathbf{X} \boldsymbol{\beta}+e
$$

where $Y$ is a vector of responses, $\mathbf{X}$ is a matrix of predictor variables, $e$ is a vector of error terms, and $\boldsymbol{\beta}$ are the model coefficients to be estimated via least squares.

The goodness-of-fit of a linear regression model can be assessed using the coefficient of determination, $R^{2}$ which describes the proportion of variance explained by the regression model. The $R^{2}$ coefficient varies between 0 and 1 values closer to 1 indicating a better fit of the model to the data. In this study, the $R^{2}$ coefficient has been used as a criteria to select predictors for the regression model when there are more than one objective correlates to the perceptual dimensions revealed through the multidimensional scaling analysis. Following this, a forward and backward stepwise regression using the resulting predictor variables with $p<0.05$ as the inclusion criterion is also conducted.

Ordinal logistic regression models have been used in the case where the predictor variable is categorical as categorical variables violate the fundamental assumptions of multiple linear regression [37]. If $Y$ is a categorical variable with $k$ ordered categories $(k=1$ to $j$ ), ordinal logistic regression models the probability $p_{i j}$ that $Y_{i}$ falls into the $j^{\text {th }}$ category or higher [see equation [2.5.3]].

$$
\ln \left(\frac{p_{i j}}{1-p_{i j}}\right)=\theta_{j}+\mathbf{x}_{i}^{T} \boldsymbol{\beta}
$$

where $\theta_{j}$ is the intercept parameter for the $j$ th category, $\mathbf{x}_{i}$ is a set of independent variables, and $\boldsymbol{\beta}$ are regression coefficients to be estimated. The coefficients for this model can be estimated via maximum likelihood.

\section{Results}

From the incomplete cyclic designs generated for each subject, an inclusion matrix $Q_{S}$ was calculated whereby:

$$
q_{s, i, j}=\left\{\begin{array}{c}
1 \text { if pair } \delta_{i, j, s} \text { is included in test } \\
0 \text { otherwise }
\end{array}\right.
$$


where $q_{s, i, j}$ is the element in the inclusion matrix $Q_{s}$ for subject $s$ and the comparison between stimulus $i$ and $j$ and $\delta_{i, j, s}$ is a judged dissimilarity.

Partial dissimilarity matrices $\tilde{D}_{s}$ were then created for each subject $s$ where:

$$
\tilde{D}_{s}=\left\{\begin{array}{c}
\delta_{i, j, s} \text { if } q_{s, i, j}=1 \\
0 \text { otherwise }
\end{array}\right.
$$

A single aggregated dissimilarity matrix $\bar{D}$ was then calculated by summing each of the partial dissimilarity matrices over the subject group and dividing by the inclusion matrices summed over the subject group:

$$
\bar{D}=\frac{\sum_{s=1}^{S} \tilde{D}_{s}}{\sum_{s=1}^{S} \tilde{Q}_{s}}
$$

Non-metric multidimensional scaling solutions were calculated for the aggregated dissimilarity matrix $\bar{D}$ in one to eight dimensions. Figure 2 illustrates the relationship between stress and the number of dimensions for these solutions. Stress is a measure of the goodness of fit of the multidimensional scaling solution to the original pairwise dissimilarities. Lower values of stress indicate a better fit between the multidimensional scaling configuration and the judged dissimilarities. As the stress is for a non-metric solution, the stress being close to zero for the eight dimensional solution implies that a nearly perfect monotonic relationship has been found between the fitted distances and measured dissimilarities, but not necessarily that the dissimilarities have been perfectly reproduced in this configuration [38. The case where a permissible ordinal transformation has been found but the relationship between the fitted distances and the original dissimilarity data is poor is known as a degenerate solution. Such degenerate solutions can be expected in non-metric multidimensional scaling when the dimensionality is high compared with the number of stimuli [35].

A commonly used criterion for the determination of how many dimensions to recover is to identify an "elbow" in the relationship between the stress and the number of dimensions. Such an "elbow" represents the point at which the addition of further dimensions does not result in a significant reduction in the stress of the recovered configuration. It can be seen in Figure 2 that there is no obvious "elbow" in the relationship between dimensionality and stress. It could be argued that a two dimensional solution would an appropriate due to the significant decrease in stress going from 
a one dimensional to a two dimensional solution. However, it would seem restrictive to limit the analysis to a two dimensional solution at this stage. As a rule of thumb, Kruskal suggests that a stress value of 0.05 represents a "good" fit 38. Therefore, a four dimensional solution will be analysed in this paper. Further thought is given to the number of dimensions required to describe the human response in section 4.2 .

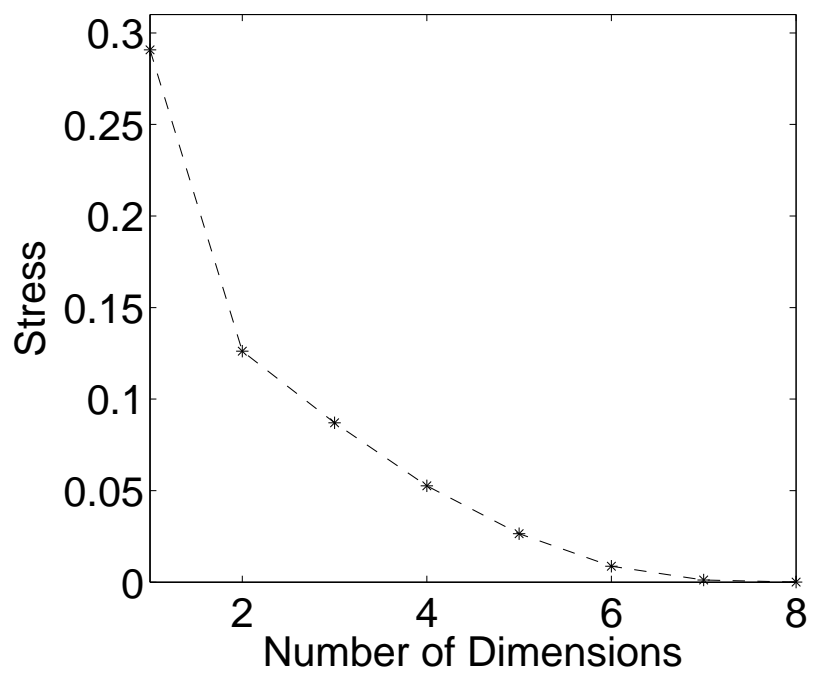

Figure 2: Scree plot showing stress as a function of number of dimensions.

Intra-subject consistency in paired comparison tests can be assessed using circular error rates [39]. A circular error is defined as occurring when a subject makes an inconsistent judgement on a triad of stimuli. For example, an inconsistency would occur if a subject were to judge stimulus A is more annoying than stimulus B, stimulus B as more annoying than stimulus $\mathrm{C}$, and stimulus $\mathrm{C}$ as more annoying than stimulus A. Figure 3 shows the circular error rates for each of the twenty-one subjects who took part in the paired comparison tests described in this paper. It can be seen from this figure that the majority of subjects were consistent in their judgements with nine subjects making no inconsistent judgements. This may be attributed to the use of an incomplete paired comparison test design as there are fewer triads of stimuli formed than in a full paired comparison test and therefore fewer opportunities for subjects to make inconsistent judgements. This suggests that, in an incomplete paired comparison test, the circular error rate may underestimate intra-subject inconsistency. Therefore, subjects exhibit- 
ing circular error rates greater than $10 \%$ were omitted from further analysis. It should be noted that this decision was made after an initial investigation into objective correlates to the perceptual dimensions calculated using data from all subjects was found to be problematic. Figure 4 shows the four

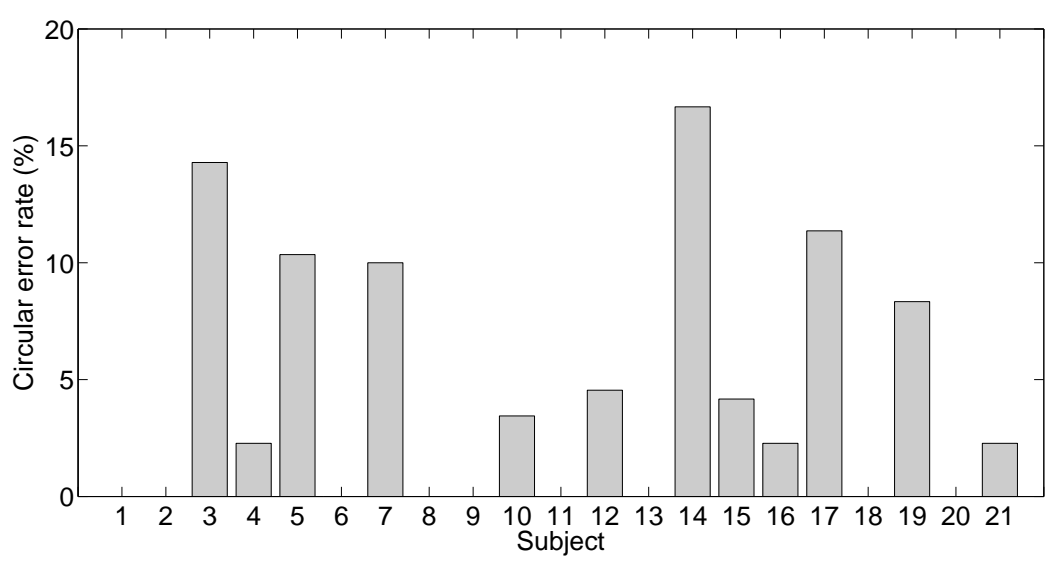

Figure 3: Circular error rate for each of the twenty-one subjects.

dimensional non-metric multidimensional scaling solution calculated after the omission of subjects 3,14 , and 17 . The fourteen points in the configurations shown in this figure represent the fourteen train vibration stimuli used in the paired comparison tests with large distances between points in the configurations representing large judged dissimilarities. It can be seen from this figure that the positions of the stimulus points are well distributed across each of the perceptual dimensions suggesting that subjects made their judgements based upon perceptual continua and did not simply categorise the stimuli.

\subsection{Paired comparisons of annoyance}

From the paired comparison of annoyance data, single figure annoyance scores were calculated for each subject using the following method:

$$
A_{i, s}=\frac{1}{N_{i}} \sum_{j \neq i} P_{j, i, s}
$$

where $A_{i, s}$ is the Perceived Annoyance Rating for subject $s$ stimulus $i, N_{i}$ is the number of times stimulus $i$ appeared in the subjective test for subject $s$ and $P_{j, i, s}$ is the paired comparison of annoyance rating for stimuli $i$ and $j$ for subject $s$. 

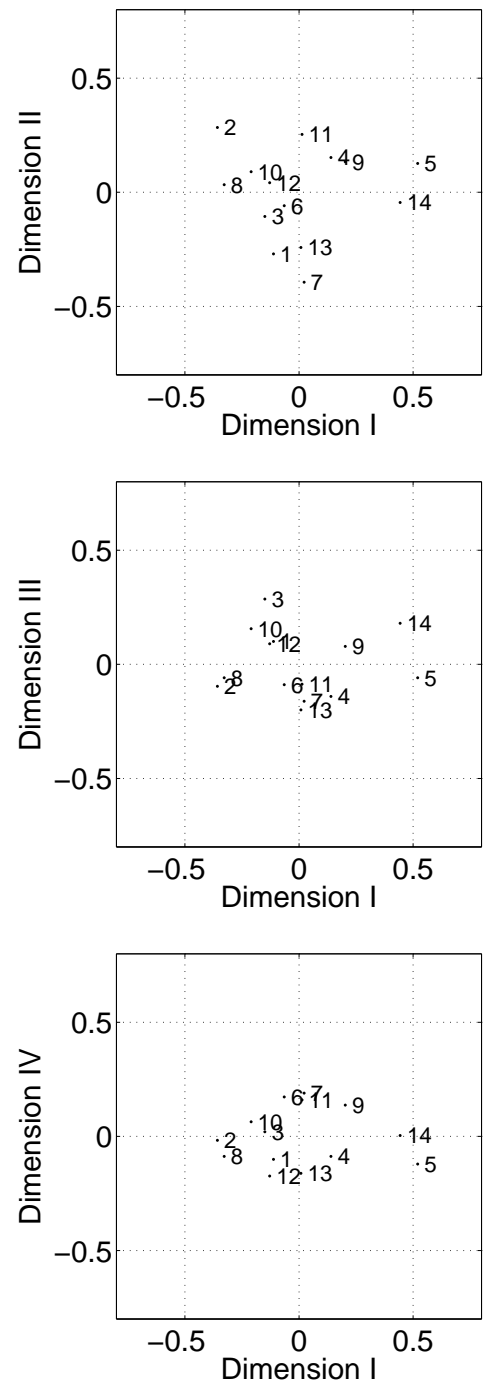

Figure 4: Four dimensional non-metric multidimensional scaling configuration calculated form the main paired comparison tests of dissimilarity. 
Calculation of single figure annoyance scores referred to in this paper as "Perceived Annoyance Ratings" using this method assumes that the perceived annoyance scores are based on an interval level psychological scale. This method has been utilised previously in the estimation of single figure merit scores in sound quality tests [40]. There are however other widely used models for the estimation of single figure scores from paired comparison data such as the Thurstone's law of categorical judgement (Case V) [41] or the Bradley-Terry-Luce [42, 43] model. To assess if $P_{j, i, s}$ is well represented by the single figure annoyance scores $A$, the matrix of annoyance judgements was reconstructed using the following relationship:

$$
\tilde{P}_{j, i}=A_{j}-A_{i}
$$

The RV coefficient, which can be interpreted as the multivariate form of the Pearson's correlation coefficient, between the matrices $P_{j, i, s}$ averaged

over all subjects and $\tilde{P}_{j, i}$ was found to be 0.93 suggesting that, although some information is lost, $P_{j, i, s}$ is well represented by the single figure annoyance scores $A$.

Figure 5 shows the Perceived Annoyance Ratings for the fourteen stimuli used in the paired comparison tests linearly averaged across the twenty-one subjects presented in their $95 \%$ confidence intervals. The narrow confidence intervals shown in this figure highlight the high inter-subject consistency which can be achieved using the method of paired comparisons. An ANOVA of the single figure Perceived Annoyance Ratings for each subject suggested that there were no significant differences between the subjects' ratings $(\mathrm{p}=$ $0.99)$. The inter-subject correlation was found to be significant in all cases $(\mathrm{p}<0.05)$.

It should again be noted that the Perceived Annoyance Ratings presented in this section are on a relative scale with an arbitrary reference point. It is clear from Figure 5, for example, that stimulus five has a greater Perceived Annoyance Rating than stimulus two. What is not clear, however, is how the stimuli would be judged on an absolute scale of annoyance.

\subsection{Categorical ratings of annoyance}

The stacked bar chart presented in Figure 6 shows the proportion of subjects rating each of the train vibration stimuli in a given annoyance category. 


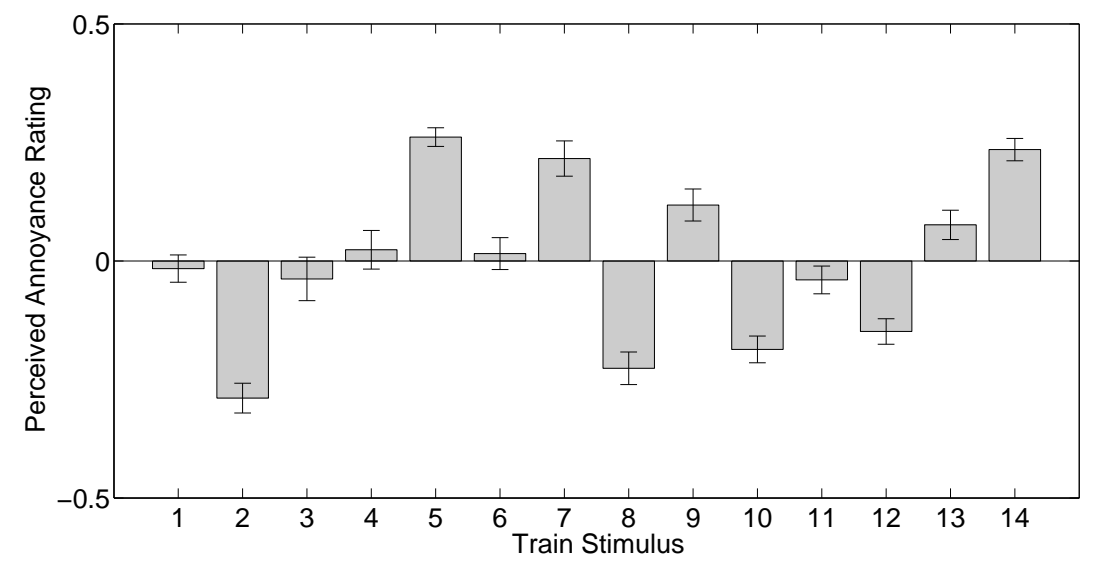

Figure 5: Perceived Annoyance Ratings for the 14 train stimuli. Values shown in their $95 \%$ confidence intervals.

The bars in this figure have been rank ordered according to the single figure Perceived Annoyance Ratings. In this figure a general trend can be observed with train stimuli exhibiting higher Perceived Annoyance Ratings being rated with higher categorical annoyance responses. Compared to the confidence intervals of the Perceived Annoyance Ratings shown in Figure 5 , there is however a large spread of different category ratings for each stimulus.

These results illustrate the advantage of paired comparison tests in terms of inter-subject variability. In a paired comparison test, there is always a reference stimulus meaning inter-subject judgements are fairly consistent. However, when making a judgement on a single stimulus on an absolute scale, the reference is likely the subject's own experience. As experience and perception varies greatly from subject to subject, so to do their responses in this type of test.

\section{Discussion}

\subsection{Interpretation of the perceptual space}

\subsubsection{Objective correlates to the perceptual dimensions}

A number of objective descriptors were calculated for each of the vibration signals as potential correlates to the perceptual dimensions revealed the multidimensional scaling analysis of the paired comparison of similarity data. 


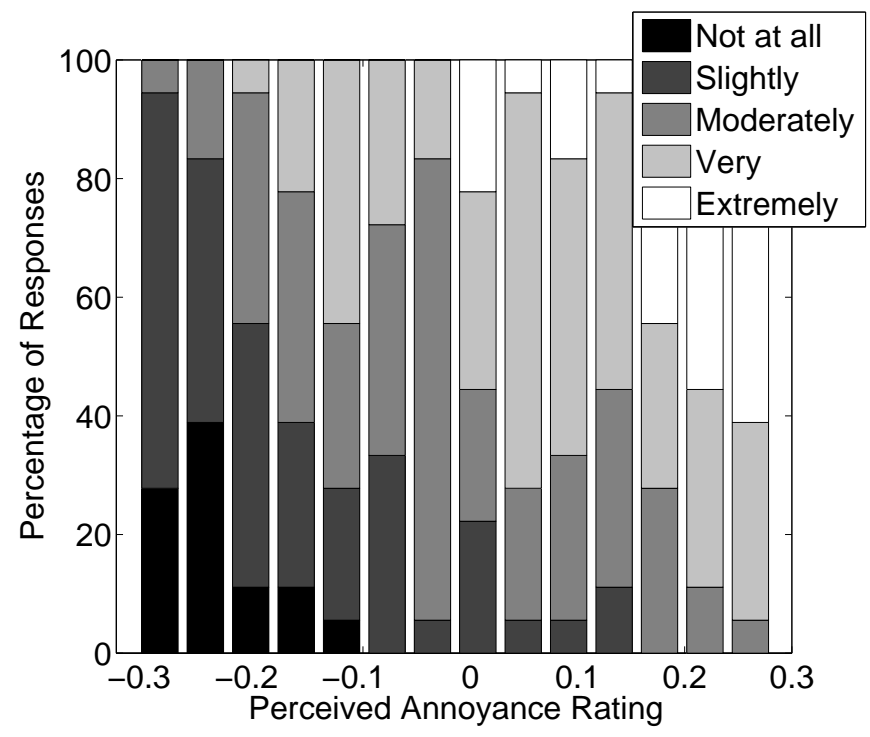

Figure 6: Categorical annoyance ratings for the 14 train stimuli.

These descriptors were calculated from measurements of acceleration made at the interface between an $81 \mathrm{~kg}$ subject and the seat cushion. Due to the compression of the seat cushion for subjects of different masses, there is some degree of uncertainty in the vibration exposure for different subjects. As measures of the energy of the stimuli, VDV, rms acceleration, peak acceleration, root mean quad (rmq) acceleration, root mean hex (rmh) acceleration, and root mean oct (rmo) acceleration were calculated. Maximum exponentially weighted running rms values were also determined with time constants of $1 \mathrm{~s}$ and $0.125 \mathrm{~s}$. These descriptors were also calculated with the application of the $W_{b}$ frequency weighting advocated in BS 6472-1:2008 [44. In addition to these descriptors of vibration energy, the 50th, 75th, 90th, and 95 th percentile of the acceleration time histories were determined. Temporal features of the stimuli were characterised through the calculation of the crest factor, the ratio between the 95th and 50th percentile, modulation depth, modulation frequency, and duration, rise time, and decay time defined by the $10 \mathrm{~dB}$ and $3 \mathrm{~dB}$ down points of the signal envelope. Statistical characteristics of the acceleration time histories were described through the sample skewness and kurtosis. Spectral centroid and the dominant frequency of the power spectral density $\left(f_{\max }\right)$ of the stimuli were calculated to characterise the frequency content. As these descriptors cover temporal, frequency, en- 
ergy, and statistical characteristics, it is assumed that the stimulus set is sufficiently characterised so as to give an indication of the nature of each of the perceptual dimensions calculated through the multidimensional scaling analysis.

\subsubsection{Dimension I}

From the considered objective vibration exposure descriptors, the first perceptual dimension revealed through the multidimensional scaling analysis was found to be significantly correlated (Pearson's correlation coefficient, $\mathrm{p}<0.05)$ to the $W_{b}$ weighted VDV, and the $W_{b}$ weighted rms, rmq, rmh, and rmo energy averages of the vibration stimuli. The correlation coefficient between this dimension and these descriptors ranged between 0.56 to 0.65. The use of higher power energy average descriptors such as the rmq, rmh, and rmo did not result in significantly higher correlations with this perceptual dimension over the more conventional rms averaging.

The frequency weighted rms and VDV descriptors were found to exhibit a stronger correlation to the first perceptual dimension than their unweighted counterparts. This suggests that the frequency content of the vibration exposure has a role in the interpretation of the first perceptual dimension. To further investigate this perceptual dimension, each of the stimuli were filtered into octave bands of centre frequency $4 \mathrm{~Hz}, 8 \mathrm{~Hz}, 16 \mathrm{~Hz}, 32 \mathrm{~Hz}$, and $64 \mathrm{~Hz}$. Peak acceleration, rms acceleration, and VDV were then determined for each octave band. Figure 7 shows the Pearson's correlation coefficient between the first perceptual dimension and these three descriptors in each octave band. It can be seen from this figure that there is a strong correlation between the first perceptual dimension and each of the calculated descriptors in the $4 \mathrm{~Hz}, 8 \mathrm{~Hz}$, and $16 \mathrm{~Hz}$ octave bands. The p-value of the correlations in each of these bands is less than 0.001. The trend in the correlations shown in Figure 7 with respect to frequency is similar to that of the apparent mass of the seated human body to vertical vibration (see, for example, 45]), vertical vibration perception thresholds for the seated position, and the $W_{b}$ and $W_{k}$ weighting curves the suggesting that the first perceptual dimension relates to vibration in the range of frequencies related to whole body vibration. To illustrate the relationships suggested by the correlation coefficients shown in Figure 7, scatter plots of the positions of each of the stimuli on the first perceptual dimension and rms acceleration in the $4 \mathrm{~Hz}, 8 \mathrm{~Hz}$, and 


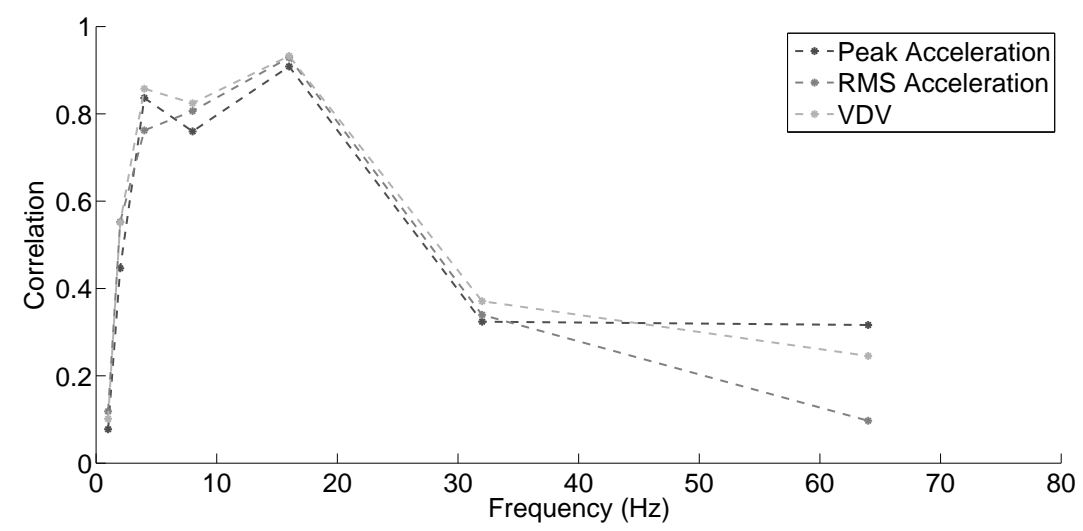

Figure 7: Pearson's correlations coefficient between the first perceptual dimension and peak acceleration, rms acceleration, and VDV in the $4 \mathrm{~Hz}, 8$ $\mathrm{Hz}, 16 \mathrm{~Hz}, 32 \mathrm{~Hz}$, and $64 \mathrm{~Hz}$ octave bands.

$16 \mathrm{~Hz}$ octave bands are presented in Figure 8. These figures confirm the relationships suggested by the correlation coefficients presented in Figure 7 .

\subsubsection{Dimension II}

From the considered objective vibration exposure descriptors, the second perceptual dimension revealed through the multidimensional scaling analysis was found to be significantly correlated (Pearson's correlation coefficient, $\mathrm{p}$ $<0.01)$ to the unweighted VDV and the unweighted rms, rmq, rmh, and rmo energy averages of the vibration stimuli. The correlation coefficient between this dimension and these descriptors ranged between -0.56 to -0.74 . Less significant correlations are also observed between this perceptual dimension and the $W_{b}$ weighted VDV and rms acceleration descriptors.

As with the first perceptual dimension, that the second perceptual dimension shows a greater degree of correlation with the unweighted VDV and rms acceleration descriptors over their $W_{b}$ weighted counterparts suggests that the frequency content of the vibration exposure has an influence upon this perceptual dimension. Figure 9 shows the Pearson's correlation coefficients between the second perceptual dimension and peak acceleration, rms acceleration, and VDV in the $4 \mathrm{~Hz}, 8 \mathrm{~Hz}, 16 \mathrm{~Hz}, 32 \mathrm{~Hz}$, and $64 \mathrm{~Hz}$ octave bands. For ease of comparison with Figure 7 , the absolute values of the correlation coefficients are shown. It can be seen from this figure that 

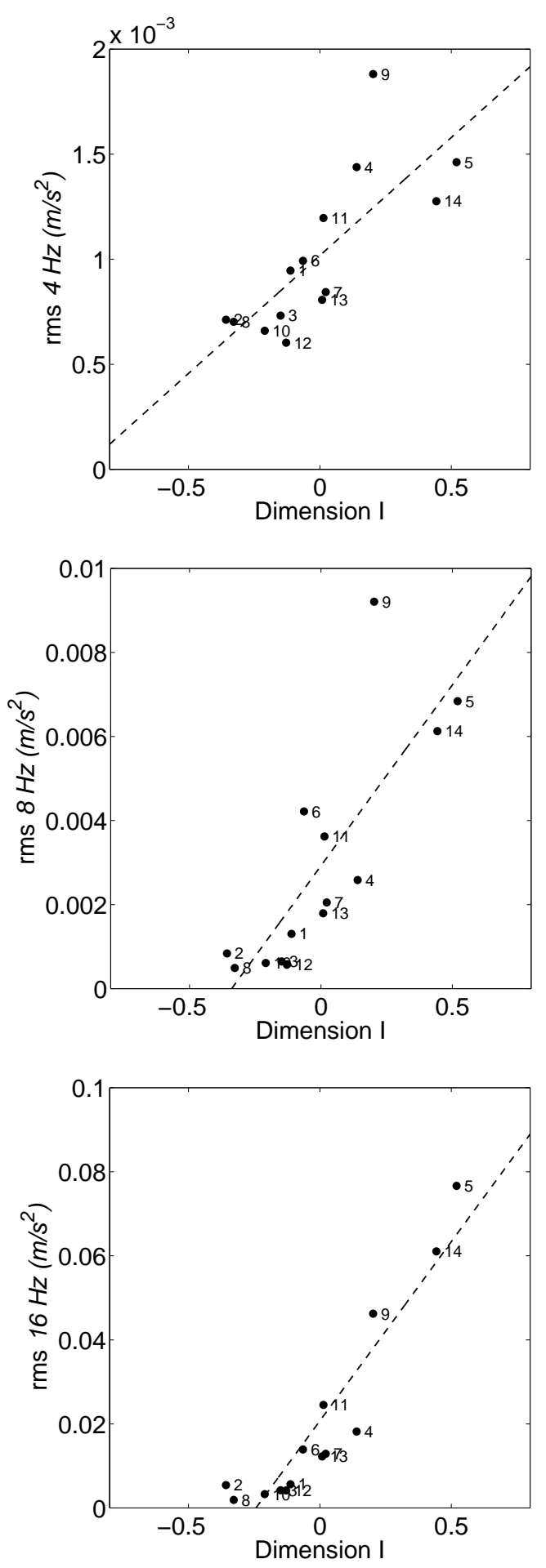

Figure 8: Relationship between the first perceptual dimension and rms acceleration in the $4 \mathrm{~Hz}, 8 \mathrm{~Hz}$, and $16 \mathrm{~Hz}$ octave bands. 
the second perceptual dimension is significantly correlated with the three descriptors in the $32 \mathrm{~Hz}$ and $64 \mathrm{~Hz}$ octave bands. This range of frequencies is generally associated with vibrotactile perception through the Pacinian and Meissner's corpuscle mechanoreceptors [46, 47]. This result, along with the findings for the first perceptual dimension, suggest that it may be appropriate to consider whole-body and vibrotactile vibration separately in the assessment of the human response to vibration, as they may contribute independently to the overall perception of vibration. To illustrate the rela-

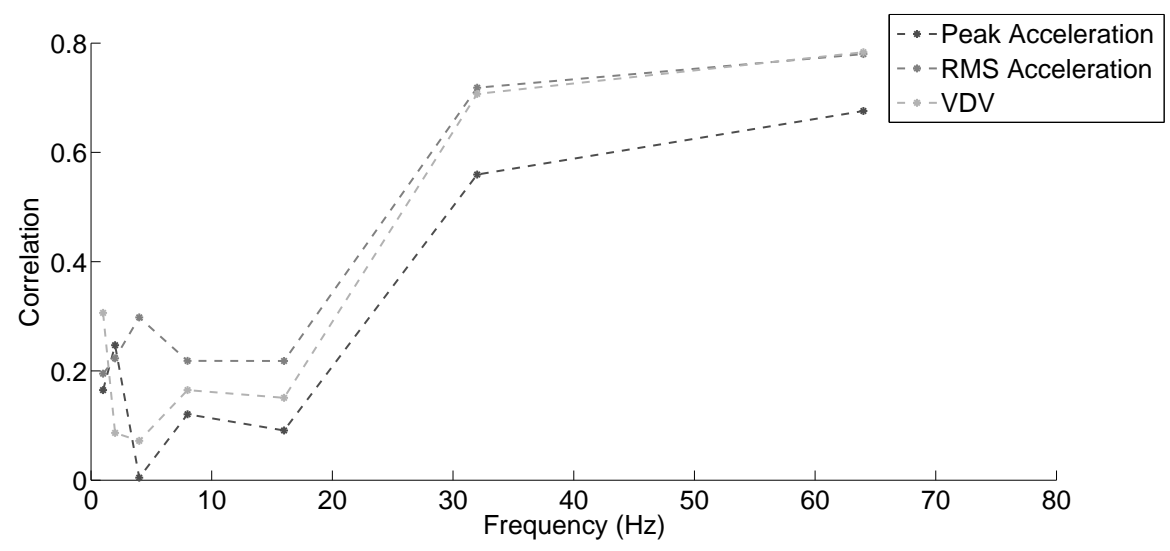

Figure 9: Pearson's correlations coefficient (absolute values) between the second perceptual dimension and peak acceleration, rms acceleration, and VDV in the $4 \mathrm{~Hz}, 8 \mathrm{~Hz}, 16 \mathrm{~Hz}, 32 \mathrm{~Hz}$, and $64 \mathrm{~Hz}$ octave bands.

tionships suggested by the correlation coefficients in Figure 9, scatter plots of the positions of the stimuli on the second perceptual dimension and rms acceleration in the $32 \mathrm{~Hz}$ and $64 \mathrm{~Hz}$ octave bands are presented in Figure 10.

\subsubsection{Dimension III}

From the considered objective vibration exposure descriptors, the third perceptual dimension revealed through the multidimensional scaling analysis was found to be significantly correlated to the crest factor and the duration of the stimuli defined by the $10 \mathrm{~dB}$ down points (Pearson's correlation coefficients of -0.58 and -0.60 respectively, $\mathrm{p}<0.05)$. Figure 11 presents scatter plots showing the relationship between the third perceptual dimension and these two descriptors. Although the correlation between this dimension and these descriptors are significant at the 0.05 level, it can be seen from this fig- 

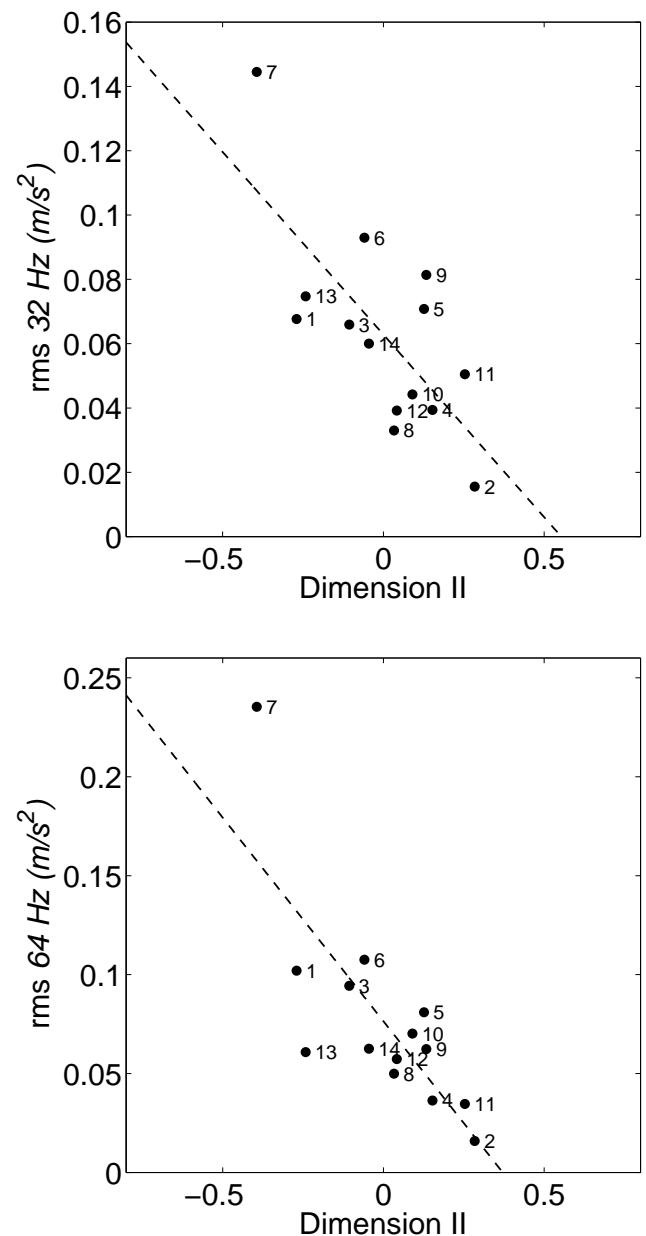

Figure 10: Relationship between the second perceptual dimension and rms acceleration in the $32 \mathrm{~Hz}$, and $64 \mathrm{~Hz}$ octave bands. 
ure that there is a large amount of scatter in these relationships suggesting that further investigation into this dimension may yield more appropriate descriptors.
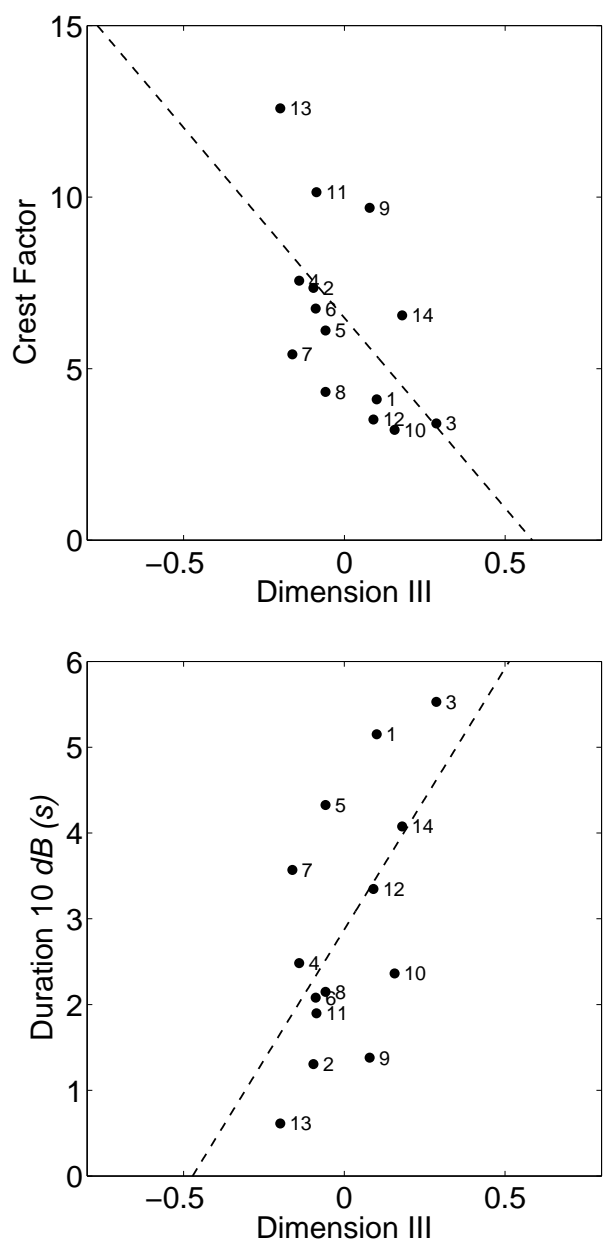

Figure 11: Relationship between the third perceptual dimension and crest factor and duration defined by the $10 \mathrm{~dB}$ down points.

\subsubsection{Dimension IV}

From the considered objective vibration exposure descriptors, the fourth perceptual dimension revealed through the multidimensional scaling analysis was found to be significantly correlated to modulation depth (Pearson's correlation coefficient of $0.79, \mathrm{p}<0.01$ ) and modulation frequency (Pearson's correlation coefficient of $-0.57, \mathrm{p}<0.05)$. The relationships between 
the fourth perceptual dimension and these descriptors are shown in Figure 12. The modulation depth and the modulation frequency are significantly correlated for the set of stimuli used in this study (Pearson's correlation coefficient of $0.7, \mathrm{p}<0.01)$ making it difficult to assess which of these descriptors is most appropriate to describe the fourth perceptual dimension. However, from the bottom pane of Figure 12 it appears that the relationship between the fourth perceptual dimension and the modulation frequency is strongly influenced by the outlier stimulus 13 , lowering the correlation.
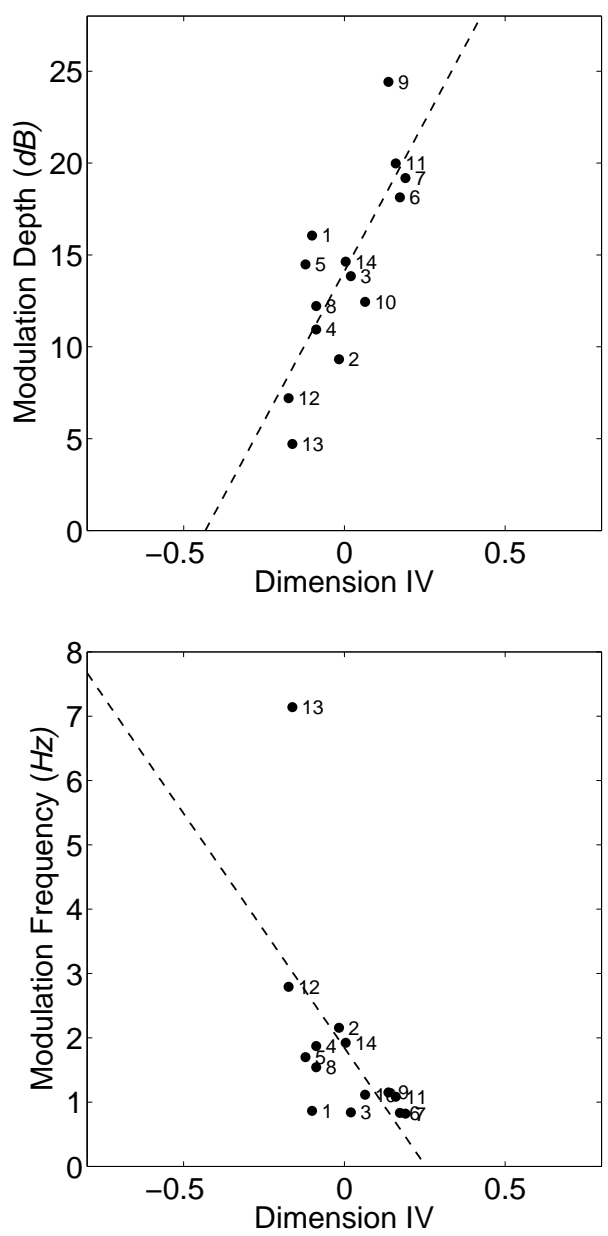

Figure 12: Relationship between the fourth perceptual dimension and modulation depth and modulation frequency. 


\subsection{Perceived annoyance models}

To investigate the relationship between the perceptual dimensions revealed through the multidimensional scaling analysis and the Perceived Annoyance Ratings calculated from the paired comparison of annoyance tests, a multiple linear regression was conducted using the Perceived Annoyance Ratings as the dependent variable and the position of the stimuli on the four perceptual dimensions as independent variables. The result of this regression is described in equation (11).

$$
A=0+0.60 D_{1}-0.34 D_{2}-0.10 D_{3}+0.23 D_{4}
$$

where $A$ is the predicted single figure Perceived Annoyance Rating and $D_{n}$ is the position of the vibration stimulus on the $n^{\text {th }}$ perceptual axis.

Apart from the intercept coefficient, which is zero, all of the coefficients in the model presented in equation (11) are statistically significant; the coefficients for dimensions I, II, and IV are significant to the 0.001 level and the coefficient for dimension III is significant to the 0.05 level. This result suggests that each of the four recovered perceptual dimensions has a significant influence upon the Perceived Annoyance Ratings. The standardised regression coefficients for the $D_{1}$ is $0.15, D_{2}$ is $-0.07, D_{3}$ is -0.02 , and $D_{4}$ is 0.03 . This suggests that the first dimension has the greatest influence on perceived annoyance, followed by the second then fourth dimension with the third dimension having the least influence. The adjusted $R^{2}$ value for this model is $0.98, \mathrm{p}<0.001$.

In the previous section, a number of potential objective correlates were found for each of the dimensions revealed through the multidimensional scaling analysis. Multiple linear regression models were calculated with every possible combination of the objective descriptors found as significant correlates to each of the perceptual dimensions. The adjusted $R^{2}$ values for the calculated models were found to range between 0.72 and 0.92 . The model exhibiting the highest value of adjusted $R^{2}$ included the rms acceleration in the $16 \mathrm{~Hz}$ and $32 \mathrm{~Hz}$ octave bands, the duration defined by the $10 \mathrm{~dB}$ down points, and the modulation frequency. The result of this regression is described by equation (12).

$$
A=-0.40+4.57 a_{16 H z}+3.18 a_{32 H z}+0.02 T_{10 d B}+0.02 f_{\text {mod }}
$$

where $A$ is the predicted single figure Perceived Annoyance Rating, $a_{16 \mathrm{~Hz}}$ and $a_{32 \mathrm{~Hz}}$ are the rms acceleration of each train event in the $16 \mathrm{~Hz}$ and 
$32 \mathrm{~Hz}$ octave band respectively, $T_{10 d B}$ is the duration of each train event defined by its $10 \mathrm{~dB}$ down points, and $f_{\text {mod }}$ is the modulation frequency of the envelope of each train event. The coefficients for the $a_{16 \mathrm{~Hz}}$ and $a_{32 \mathrm{~Hz}}$ terms are significant to the 0.001 level and the coefficients for the $T_{10 d B}$ and $f_{\text {mod }}$ terms are significant to the 0.05 level.

Figure 13 shows a comparison between the Perceived Annoyance Ratings measured through the perpetual testing and the Perceived Annoyance Ratings predicted using equation $(12)$. The adjusted $R^{2}$ value for this model is $0.92, \mathrm{p}<0.001$.

If a regression model were derived using only the weighted rms acceleration, as recommend in ISO 2631 - 1 [31], as the independent variable the adjusted $R^{2}$ value for the resulting model would be 0.73 . Similarly, a model using only the vibration dose value as an independent variable results in an adjusted $R^{2}$ of 0.79 . This suggests that the Perceived Annoyance Rating model in equation 12 accounts for $19 \%$ more variance in the annoyance ratings than a model using only the weighted rms acceleration and $13 \%$ more of the variance than a model using only the vibration dose value.

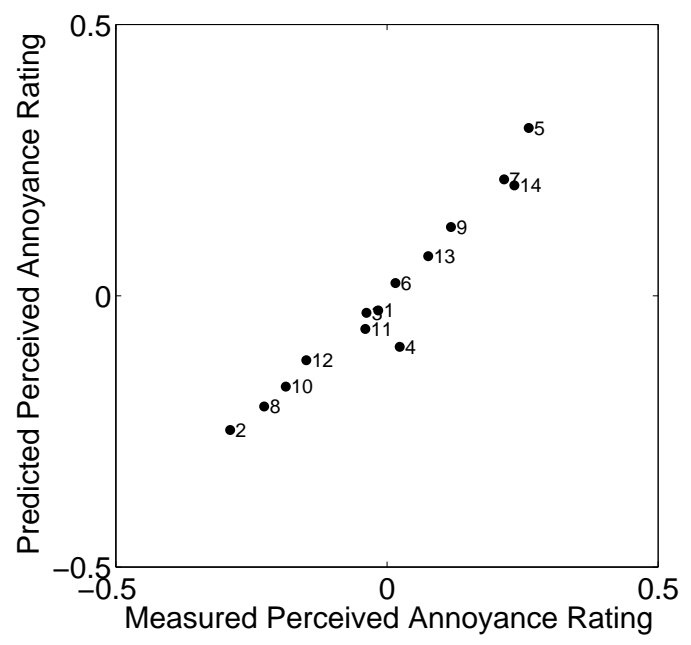

Figure 13: Comparison between the measured Perceived Annoyance Ratings and those predicted using the model in equation 12 .

To explore the trade-off between reducing the number of predictor variables in the model described by equation $(12)$ and the amount of variance explained by the model, a stepwise regression was conducted. The criterion for the inclusion of a predictor variable in the model is that the estimated 
$\beta$ coefficient must have a $\mathrm{p}$-value of less than 0.05 . The stepwise regression resulted in dropping the $T_{10 d B}$ and $f_{\text {mod }}$ terms from the model. The resulting adjusted $R^{2}$ for this model is 0.88 confirming that the reduced model describes a similar amount of variance as the full model. That the $a_{16 \mathrm{~Hz}}$ and $a_{32 \mathrm{~Hz}}$ terms both contribute significantly to the annoyance response further supports the hypothesis put forward in section 4.1.3 that the vibration in these two frequency regions should be considered separately in the assessment of human response.

In the model relating the positions of the stimuli on the four perceptual dimensions to the Perceived Annoyance Ratings [see equation [11]], it can be noted that each of the coefficients in the model reached statistical significance. This suggests that further work is needed to find objective correlates which better describe the third and fourth perceptual dimensions.

\subsubsection{Categorical annoyance model}

As discussed in section 3.1, the Perceived Annoyance Ratings calculated from the paired comparison of annoyance tests are relative to the group of stimuli on which they were judged and are on an arbitrary scale. That is to say, although a greater Perceived Annoyance Rating implies greater annoyance, it is unknown what the absolute rating of that annoyance is. From the categorical annoyance ratings, each train stimulus was attributed a single figure rating by taking the mode of the annoyance ratings for each stimulus. Figure 14 shows the relationship between the Perceived Annoyance Ratings and these categorical annoyance ratings. The Spearman's correlation coefficient between these two variables is 0.93 ( $\mathrm{p}<0.0001)$.

An ordinal logistic regression model was calculated to relate the Perceived Annoyance Ratings to the categorical annoyance ratings. The results of this model are shown in Figure 15. The curves in this figure indicate the probability of a train with a given Perceived Annoyance Rating being rated in a certain annoyance category.

\subsection{Validation of Perceived Annoyance Rating model}

As a validation of the relative Perceived Annoyance Rating model, the model described in equation 11 was used to predict the single figure annoyance ratings measured in a pilot test which was conducted previous to this study. 


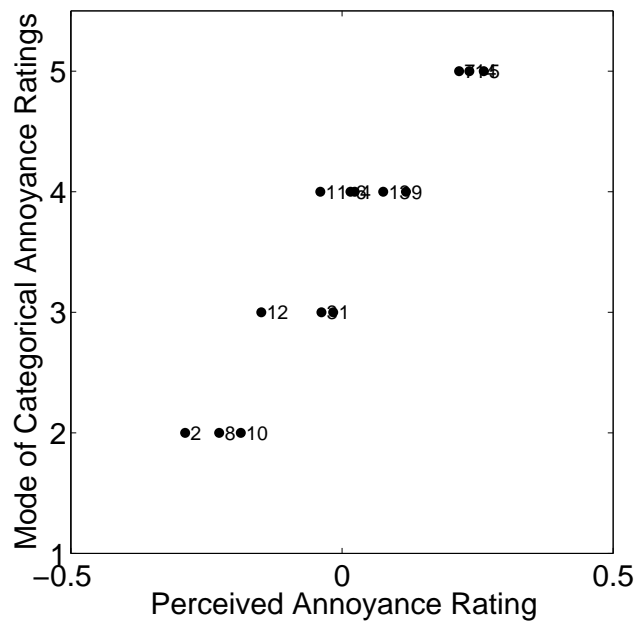

Figure 14: Relationship between Perceived Annoyance Ratings and categorical annoyance ratings.

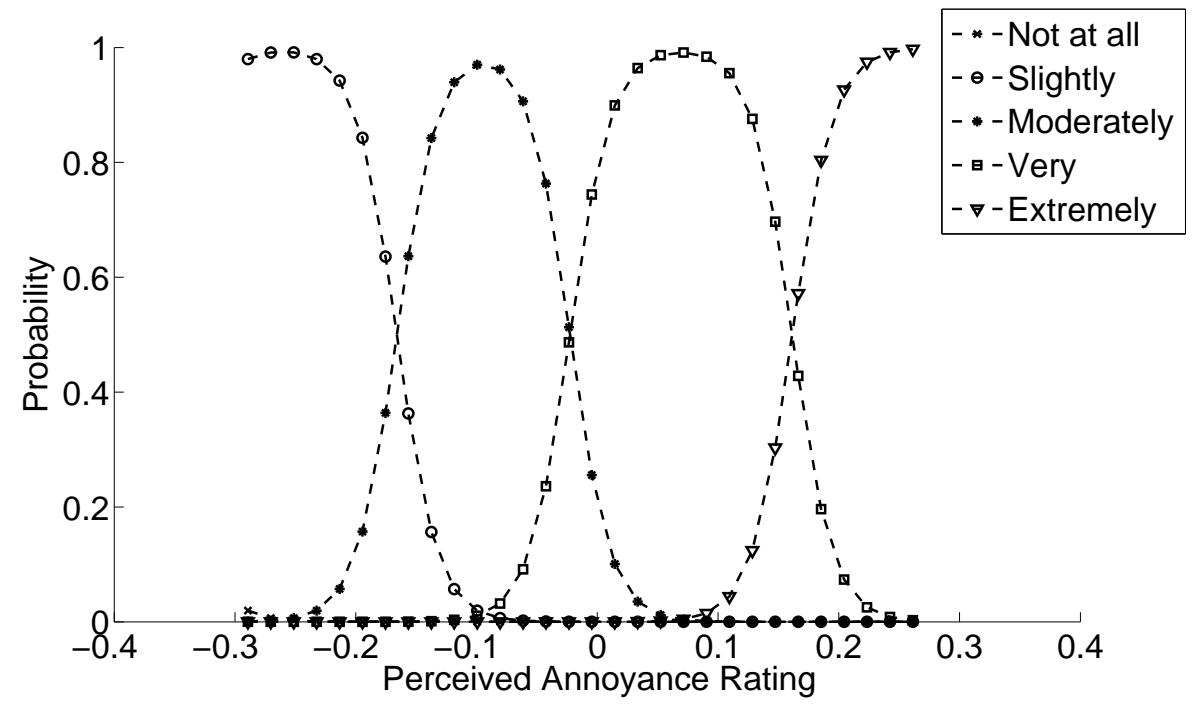

Figure 15: Probability of a railway vibration event with a given Perceived Annoyance Rating being rated in a certain annoyance category on a fivepoint semantic scale. 
These data are therefore independent of the data that were used to derive the model. Figure 16 shows a comparison between the annoyance ratings measured in this pilot test and the ratings predicted using the model in equation (11). The Pearson's correlation coefficient between the measured and predicted annoyance ratings shown in this figure is 0.91 ( $\mathrm{p}<0.0001)$. As the Perceived Annoyance Ratings are a relative measure of annoyance and are therefore arbitrary and dependent upon the set of stimuli on which they were judged, the absolute values of the annoyance ratings shown in this figure differ. The predicted annoyance values are higher than the measured because the stimuli used in the pilot test were of a higher magnitude that those used in the tests described in this paper. It can however be seen that there is good agreement in the trend of the measured and predicted values suggesting that the model was successfully able to predict the relative perceived annoyance ratings.

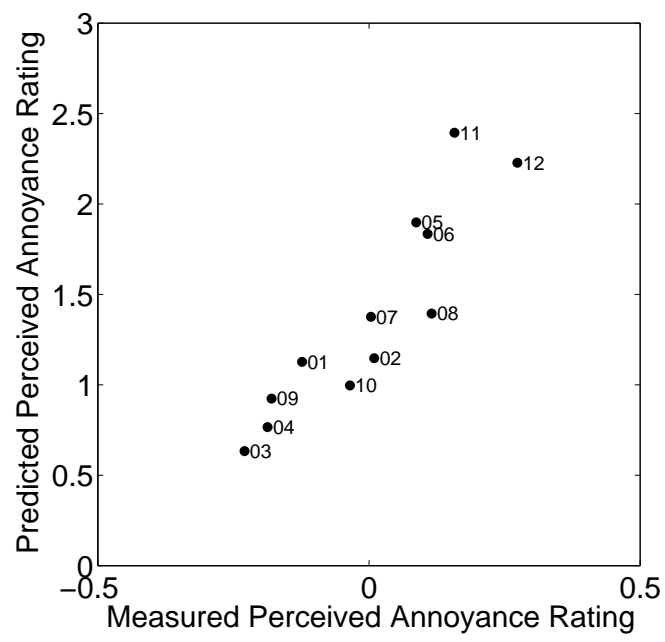

Figure 16: Comparison between Perceived Annoyance Ratings measured in a pilot test using a different set of vibration stimuli and those predicted using the model in equation (12).

\section{Conclusions}

This paper presents the results of a paired comparison test designed to investigate the perception of railway induced groundborne vibration. Multidimensional scaling analysis of the data from this experiment has shown 
that the perception of whole body vibration from railway activities is multidimensional in nature. Investigation of the perceptual space resulting from this analysis has shown that perception of railway induced vibration is dependent on up to four perceptual dimensions which relate to energy in the $16 \mathrm{~Hz} 1 / 3$ octave band, energy in the $32 \mathrm{~Hz} 1 / 3$ octave band, the duration of the train passage, and the modulation frequency of the envelope of the signal. It is shown that these perceptual dimensions can be linearly related to single figure measures of annoyance which in turn can be related to absolute category ratings of annoyance.

The main limitation of the work is the size of the subject group; previous studies into the perception of sound and vibration have found inter-subject groupings from which classes of subject could be identified [48]. Due to the size of the subject group in the present study, such groupings may not be apparent. However, analysis of the paired comparison of annoyance ratings presented in section 3.1 suggested that there were no significant differences across the subject group in this study. The correlation between the third and fourth perceptual dimensions and the objective features is lower than in studies using paired comparisons and MDS to study the perception of sound. Therefore, further investigation is needed into descriptors relevant to these dimensions.

\section{Acknowledgement}

This work was supported by the Engineering and Physical Sciences Research Council [EP/P505089/1] and the Department of Environment, Food and Rural Affairs, UK.

The archived file is not the final published version of the article

Woodcock, J., A. T. Moorhouse, and D. C. Waddington. "A multidimensional evaluation of the perception and annoyance caused by railway induced groundborne vibration." Acta Acustica united with Acustica 100.4 (2014): 614-627.

(C)2014 S. Hirzel Verlag/European Acoustics Association

The definitive publisher-authenticated version is available online at http: //www. ingentaconnect.com/content/dav/aaua

http://dx.doi.org/10.3813/AAA.918741 
Readers must contact the publisher for reprint or permission to use the material in any form

\section{References}

[1] European Commission: Roadmap to a Single European Transport Area - Towards a competitive and resource efficient transport system. Brussels, 2011.

[2] E. Öhrström, A. Skånberg: A field survey on effects of exposure to noise and vibration from railway traffic, part I: Annoyance and activity disturbance effects. Journal of Sound and Vibration 193 (1996) 39-47.

[3] R. Klæboe, I. Turunen-Rise, L. Hårvik, C. Madshus: Vibration in dwellings from road and rail trafficPart II: exposure-effect relationships based on ordinal logit and logistic regression models. Applied Acoustics 64 (2003) 89-109.

[4] T. Yano: Community response to Shinkansen noise and vibration: a survey in areas along the Sanyo Shinkansen Line. In the proceedings of Forum Acusticum, Budapest, 2005, 1837-1841.

[5] J. Zapfe, H. Saurenman, S. Fidell: Ground-Borne Noise and Vibration in Buildings Caused by Rail Transit. TCRP Project D-12, 2009.

[6] D.C. Waddington, J. Woodcock, J. Condie, E. Peris, G. Sica, A. Moorhouse, A. Steele: Human Response to Vibration in Residential Environments. Journal of the Acoustical Society of America 135 (2014) $182-193$.

[7] A. Gidlöf-Gunnarsson, M. Ögren, T. Jerson, E. Ohrström: Railway noise annoyance and the importance of number of trains, ground vibration, and building situational factors. Noise and Health 14 (2012) 190-201.

[8] C. Marquis-Favre, E. Premat: Noise and its Effects A Review on Qualitative Aspects of Sound. Part I: Notions and Acoustic Ratings. Acta Acustica united with Acustica 91 (2005) 613-625. 
[9] C. Marquis-Favre: Noise and its Effects A Review on Qualitative Aspects of Sound. Part II: Noise and Annoyance. Acta Acustica united with Acustica 91 (2005) 626-642.

[10] J. Leatherwood, T. Dempsey: Psychophysical Relationships Characterizing Human Response to Whole-Body Sinusoidal Vertical Vibration. NASA Technical Note TN D-8188 (1976).

[11] M. Morioka, M. J. Griffin: Absolute thresholds for the perception of fore-and-aft, lateral, and vertical vibration at the hand, the seat, and the foot. Journal of Sound and Vibration 314 (2008) 357-370.

[12] M. Morioka, M. J. Griffin: Magnitude-dependence of equivalent comfort contours for fore-and-aft, lateral, and vertical vibration at the foot for seated persons. Journal of Sound and Vibration 329 (2010) 2939-2952.

[13] H. V. C. Howarth, M. J. Griffin: Human response to simulated intermittent railway-induced building vibration. Journal of Sound and Vibration 120 (1988) 413-420.

[14] H. V. C. Howarth, M. J. Griffin: Subjective reaction to vertical mechanical shocks of various waveforms. Journal of Sound and Vibration 147 (1991) 395-408.

[15] H. V. C. Howarth, M. J. Griffin: Subjective response to combined noise and vibration: summation and interaction effects. Journal of Sound and Vibration 143 (1990) 443-454.

[16] H. V. C. Howarth, M. J. Griffin: The relative importance of noise and vibration from railways. Applied Ergonomics 21 (1990) 129-134.

[17] H. V. C. Howarth, M. J. Griffin: The annoyance caused by simultaneous noise and vibration from railways. Journal of the Acoustical Society of America 89 (1991) 2317-2323.

[18] P. J. Lee, M. J. Griffin: Combined effect of noise and vibration produced by high-speed trains on annoyance in buildings. Journal of the Acoustical Society of America 133 (2013) 2126-2135.

[19] S. McAdams, S. Winsberg, S. Donnadieu, G. Soete, J. Krimphoff: Perceptual scaling of synthesized musical timbres: Common dimensions, 
specificities, and latent subject classes. Psychological research 58 (1995) 177-192.

[20] Ö. Axelsson, M. E. Nilsson, B. Berglund: A principal components model of soundscape perception. Journal of the Acoustical Society of America 128 (2010) 2836-2846.

[21] R. Guski: Psychological methods for evaluating sound quality and assessing acoustic information. Acta Acustica united with Acustica 83 (1997) $765-774$.

[22] E. Parizet, M. Amari, V. Nosulenko: Vibro-acoustical comfort in cars at idle: human perception of simulated sounds and vibrations from 3and 4-cylinder diesel engines. International Journal of Vehicle Noise and Vibration 3 (2007) 143-156.

[23] W. Torgerson: Multidimensional scaling: I. Theory and method. Psychometrika 17 (1952) 401-419.

[24] J. Grey: Multidimensional perceptual scaling of musical timbres. Journal of the Acoustical Society of America 61 (1977) 1270-1277.

[25] M. Schroeder, D. Gottlob, K. Siebrasse: Comparative study of European concert halls: Correlation of subjective preference with geometric and acoustic parameters. Journal of the Acoustical Society of America 56 (1974) 1195-1201.

[26] P. Susini, S. McAdams, S. Winsberg: A multidimensional technique for sound quality assessment. Acta Acustica united with Acustica 85 (1999) 65-656.

[27] G. Lemaitre, P. Susini: The sound quality of car horns: a psychoacoustical study of timbre. Acta Acustica united with Acustica 93 (2007) $457-468$.

[28] E. Parizet, E. Guyader, V. Nosulenko: Analysis of car door closing sound quality. Applied Acoustics 69 (2008) 12-22.

[29] G. Lemaitre, P. Susini: The sound quality of car horns: Designing new representative sounds. Acta Acustica united with Acustica 95 (2009) 356-372. 
[30] G. Sica, E. Peris, J. Woodcock, A. Moorhouse, D. Waddington: Design of measurement methodology for the evaluation of human exposure to vibration in residential environments. Science of the Total Environment In Press (2013).

[31] International Organisation for Standardization: ISO 2631-1:1997 Mechanical vibration and shock - Evaluation of human exposure to wholebody vibration - Part 1: General requirements (1997).

[32] I. Spence, D. Domoney: Single subject incomplete designs for nonmetric multidimensional scaling. Psychometrika 39 (1974) 469-490.

[33] T. Cox, M. Cox: Multidimensional scaling. Chapman \& Hall/CRC, 2001.

[34] A. Coxon, P. Davies: The user's guide to multidimensional scaling. Ashgate Publishing Limited, 1982.

[35] I. Borg, P. Groenen: Modern multidimensional scaling: Theory and applications. Springer Verlag, 2005.

[36] S. Weisberg: Applied linear regression: Second edition. Wiley, 1985.

[37] A. Agresti: Categorical data analysis. Wiley, 1990.

[38] J. Kruskal: Multidimensional scaling by optimizing goodness of fit to a nonmetric hypothesis. Psychometrika 29 (1964) 1-27.

[39] E. Parizet: Paired comparison listening tests and circular error rates. Acta Acustica united with Acustica 88 (2002) 594-598.

[40] E. Parizet, N. Hamzaoui, G. Sabatié: Comparison of some listening test methods: a case study. Acta Acustica united with Acustica 91 (2005) 356-364.

[41] L. Thurstone: A law of comparative judgment. Psychological review 34 (1927) 273-286.

[42] R. Bradley, M. Terry: Rank analysis of incomplete block designs: I. The method of paired comparisons. Biometrika 34 (1952) 324-345.

[43] R. Luce: Individual choice behaviour: A theoretical analysis. Wiley, New York, 1959. 
[44] British Standards Institute: BS 6472-1:2008 Guide to evaluation of human response to vibration in buildings $(1 \mathrm{~Hz}$ to $80 \mathrm{~Hz})$. Vibration sources other than blasting.

[45] T. E. Fairley, M. J. Griffin: The apparent mass of the seated human body: vertical vibration. Journal of Biomechanics 22 (1989) 81-94.

[46] E. Kandel, J. Schwartz, T. Jessell: Principles of neural science. McGraw-Hill, 2000.

[47] M. Gandhi, R. Sesek, R. Tuckett, S. Bamberg: Progress in Vibrotactile Threshold Evaluation Techniques: A Review. Journal of Hand Therapy 24 (2011) 240-256.

[48] E. Parizet, J. Brocard, B. Piquet : Influence of noise and vibration to comfort in diesel engine cars running at idle. Acta Acustica united with Acustica 90 (2004) 987-993. 